\title{
SEMILOCAL SEMIGROUP RINGS
}

\author{
by JAN OKNIŃSKI
}

(Received 28 July, 1982)

1. Introduction. Semilocal and related classes of group rings have been investigated by many authors (cf. [10]). In particular, the following results have been obtained.

TheOREM $\mathrm{A}[4,10]$. Let $K$ be a field and $G$ a group.

(i) If ch $K=0$, then $K[G]$ is semilocal if and only if $G$ is finite.

(ii) If ch $K=p>0$ and $G$ is locally finite, then $K[G]$ is semilocal if and only if $G$ contains a p-subgroup of finite index.

In the case of semigroup rings some stronger conditions have been studied. Munn examined the semisimple artinian situation [6]. Zelmanov showed that if $K[G]$ is artinian then $G$ must be finite [11].

The purpose of the present paper is to characterize semilocal semigroup rings $K[G]$ be means of the properties of the semigroup $G$. It is done in the cases listed in Theorem A.

Fundamental definitions and properties of semigroups and group rings may be found in $[1,10]$. In what follows $K$ will be a field and $G$ a semigroup. If $G$ contains a unity then we shall denote by $G_{1}$ the subgroup of invertible elements in $G$ and put $G_{0}=G \backslash G_{1}$. If $G$ has no unity, then $G_{0}=G$. Let us notice that if $G$ contains a unity and $K[G]$ is semilocal, then $K[G]$ is von Neumann finite [2] and so $G_{0}$ is an ideal in $G$. The set of idempotents of $G$ will be denoted by $E(G)$. If $A$ is a ring $J(A)$ will denote the Jacobson radical of $A$. For a semilocal ring $A$ we use $n_{\mathrm{A}}$ for the length of $A$-module $A / J(A)$.

The starting point for our considerations is the following result.

Theorem B $[4,9]$. Assume that $K[G]$ is semilocal. Then

(i) $G$ is torsion,

(ii) $G$ is locally finite if $\operatorname{ch} K=0$.

2. Some necessary conditions. If the assumptions of Theorem $A$ hold, then the $K$-algebra $K[G]$ is finite dimensional modulo $J(K[G])$. We will prove the same in the semigroup case.

Theorem 1. Assume that $K[G]$ is semilocal. Then $K[G] / J(K[G])$ is finite dimensional over $K$ if either of the following holds:

(i) $\operatorname{ch} K=0$,

(ii) $G$ is locally finite.

Proof. We will proceed by induction on the length $n_{K[G]}$ of the $K[G]$-module $K[G] / J(K[G])$. If $n_{K[G]}=1$ then $K[G]$ is local and $K[G] / J(K[G]) \simeq K$. Assume that $N>1$ is such that the assertion holds for $K[G]$ with $n_{K[G]}<N$. Let us consider two cases.

Case $I$. G contains a unity. Let $M$ be a maximal ideal in $K[G]$. If $M \supset K\left[G_{0}\right]$, then

Glasgow Math. J. 25 (1984) 37-44. 
$K[G] / M$ is a simple image of the algebra $K[G] / K\left[G_{0}\right] \simeq K\left[G_{1}\right]$ and thus it is of finite dimension over $K$ by Theorem A. If $M \not K\left[G_{0}\right]$, then $K[G] / M \simeq K\left[G_{0}\right] /\left(M \cap K\left[G_{0}\right]\right)$. Moreover $n_{K\left[G_{0,}\right]}<n_{K[G]}$ since the elements of $K\left[G_{0}\right] / J\left(K\left[G_{0}\right]\right)$ cannot be invertible in $K[G] / J(K[G])$. Thus, by the induction hypothesis, $K[G] / M$ is also of finite dimension over $K$, which completes the proof in this case.

Case II. $G$ has no unity. Let $M$ be a maximal ideal in $K[G]$. Then there exists $e \in E(G)$ such that $e \notin M$. (Suppose not. Then $G$ would be nil modulo $M$ by Theorem B and hence nilpotent modulo $M$ since $K[G] / M$ is artinian $[2,17.19]$. This is impossible while $M$ is a prime ideal and $G$ is irreducible in $K[G] / M$.) It is well known that $n_{K[e G e]} \leqslant n_{K[G]}$. Now, it follows from Case I that $K[e G e]$ is finite dimensional modulo its radical. Thus, by the choice of $e, K[G] / M$ is finite dimensional. Since $M$ was an arbitrary maximal ideal in $K[G]$, then the dimension of $K[G] / J(K[G])$ over $K$ is also finite.

Now we are in a position to reduce the case of semilocal semigroup algebras with arbitrary coefficients to the case where the coefficients are in a field.

Corollant 1. Let $A$ be a $K$-algebra. Assume that $G$ is locally finite. Then $A[G]$ is semilocal if and only if so are the algebras $A$ and $K[G]$.

Proof. Since $A[G] \approx A \otimes_{K} K[G]$, then the assertion follows from Theorem 1 and [3, Theorem 2.3].

We are now going to establish some connections between the characteristic zero case and the cases of positive characteristics.

Lemma 1. Assume that $G$ is locally finite. Let $a_{1}, \ldots, a_{k} \in \mathbf{Z}[G]$ be $\mathbf{Q}$-linearly dependent modulo $J(\mathbf{Q}[G]) \cap \mathbf{Z}[G]$. If $p$ is a prime number and $\bar{a}_{i}$ is the image of $a_{i}$ under the natural epimorphism $\mathbf{Z}[G] \rightarrow F_{p}[G]$, then $\bar{a}_{1}, \ldots, \bar{a}_{k}$ are $F_{\mathrm{p}}$-linearly dependent modulo $J\left(F_{\mathrm{p}}[G]\right)$.

Proof. We may assume that $b=\sum_{i=1}^{k} n_{i} a_{i} \in J(\mathbf{Q}[G])$ for some integers $n_{1}, \ldots, n_{k}$, not all divisible by $p$. Since $J(\mathbf{Q}[G])$ is nil, then $b \in J(\mathbf{Q}[G]) \cap \mathbf{Z}[G] \subset J(\mathbf{Z}[G])$. Thus $\bar{b}=\sum_{i=1}^{k} \bar{n}_{i} \bar{a}_{i} \in$
$J\left(F_{p}[G]\right)$ and there exists $i$ such that $\bar{n}_{i} \neq 0$.

For an arbitrary field $K$ let us introduce a relation $\sim_{K}$ putting $g \sim_{K} h$ if and only if $g-h \in J(K[G])$ for $g, h \in G$. Some interesting properties of this congruence are contained in the following result.

Lemma 2. Assume $K[G]$ is semilocal. Then

(i) $\sim_{K}$ is identical with $\sim_{K_{0}}$ for the prime subfield $K_{0}$ in $K$ and

(ii) in the case when $K=\mathbf{Q}$ we have

(a) if $g \sim_{K} h$ then $g \sim_{F_{\mathrm{p}}} h$ for any prime $p$ and

(b) there exist prime numbers $p_{1}, \ldots, p_{n}$ such that $\sim_{K}=\bigcap_{i=1}^{n} \sim_{F_{i}}$. 

see [8].

Proof. (i) follows from the fact that $J(K[G]) \simeq K \otimes_{K_{1}} J\left(K_{0}[G]\right)$ for semilocal $K[G]$

(ii)(a) Since $G$ is locally finite by Theorem B, (ii)(a) follows as in Lemma 1.

(ii)(b) Assume that $g-h \notin J(\mathbf{Q}[G])$ for some $g, h \in G$. Then there exists $a=\sum n_{i} g_{i} \in$ $\mathbf{Z}[G]$ such that $b=(g-h) a$ is not nilpotent. Let $G^{\prime}$ be the subsemigroup generated by the support of $b$. Then $\left|G^{\prime}\right|<\infty$. Now for $p>\left(2 \sum\left|n_{i}\right|\right)^{\left|G^{\prime}\right|}$ we get $\bar{b}^{\left|G^{\prime}\right|} \neq 0$ for the image $\bar{b}$ of $b$ under the natural epimorphism $\mathbf{Z}[G] \rightarrow F_{\mathrm{p}}[G]$. Thus $\bar{b}=(g-h) \bar{a}$ is not nilpotent since nilpotents in $F_{\mathrm{p}}\left[G^{\prime}\right]$ have degrees at most $\left|G^{\prime}\right|$, and so $g-h \notin J\left(F_{p}[G]\right)$. Hence $\sim_{\mathbf{Q}}=$ $\bigcap \sim F_{v}$.

For any prime $p$ let us consider the commuting diagram given by the natural epimorphisms.

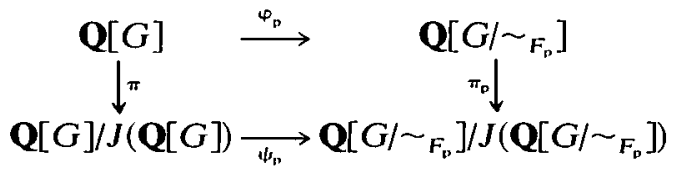

If $g-h \in J\left(\mathbf{Q}\left[G / \sim_{F_{\mathrm{p}}}\right]\right)$ for some $g, h \in G / \sim_{F_{\mathrm{p}}}$, then by (a) $g-h \in J\left(F_{\mathrm{p}}\left[G / \sim_{F_{\mathrm{p}}}\right]\right)$, which implies $g=h$. Hence the restriction of $\pi_{p}$ to $G / \sim_{F_{\mathrm{p}}}$ is a semigroup isomorphism. Since $\mathbf{Q}[G]$ :s semilocal, there exist finitely many different kernels of homorphisms of the form $\psi_{\mathrm{p}} \pi$. However, if $\sim_{F_{\mathrm{r}}}, \sim_{F_{4}}$ are distinct congruences, then the kernels of the homomorphisms $\psi_{p} \pi, \psi_{\mathrm{q}} \pi$ are different. In fact, if for example there exist $x, y \in G$ such that $x \sim_{F_{\mathrm{v}}} y$, $x \psi_{F_{\mathrm{q}}} y$, then $x-y \in \operatorname{ker} \pi_{\mathrm{p}} \varphi_{\mathrm{p}}=\operatorname{ker} \psi_{\mathrm{p}} \pi$ and $x-y \notin \operatorname{ker} \pi_{\mathrm{q}} \varphi_{\mathrm{q}}=\operatorname{ker} \psi_{\mathrm{q}} \pi$. Hence there exist finitely many congruences of the form $\sim_{F_{p}}$ in $G$ and $\sim_{\mathbf{Q}}=\bigcap_{i=1}^{n} \sim_{F_{p_{i}}}$ for some primes $p_{1}, \ldots, p_{n}$.

THEOREM 2. Assume ch $K=0$ or $G$ is locally finite. If $K[G]$ is semilocal, then the semigroup $G / \sim_{K}$ is finite.

Proof. Let $K_{0}$ be the prime subfield in $K$. Then $K_{0}[G]$ is semilocal (cf. [3]) and so $K_{0}[G] / J\left(K_{0}[G]\right)$ is finite dimensional over $K_{0}$ by Theorem 1 . If ch $K=p>0$, then $G / \sim_{K}=G / \sim_{F_{\mathrm{p}}}$ is finite since it is embeddable into the finite ring $F_{\mathrm{p}}[G] / J\left(F_{\mathrm{p}}[G]\right)$. In the case of characteristic zero it follows from Lemma 2 that

$$
G / \sim_{K}=G /\left(\bigcap_{i=1}^{n} \sim_{F_{p_{i}}}\right) \subset \prod_{i=1}^{n}\left(G / \sim_{F_{p_{i}}}\right)
$$

for some primes $p_{i}$. Now, by Theorem 1 , Theorem B and Lemma $1, F_{p}[G] / J\left(F_{p}[G]\right)$ is finite dimensional over $F_{p}$ and, as above, $G / \sim_{F_{n_{i}}}$ is finite. Thus $G / \sim_{K}$ is also finite.

Since we have natural epimorphisms $K[G] \rightarrow K\left[G / \sim_{K}\right] \rightarrow K[G] / J(K[G])$, the above result shows that $K[G] / J(K[G])$ is a homomorphic image of a semigroup ring of a finite semigroup. Thus Theorem 2 may be regarded as a strengthened version of Theorem 1 .

Let us notice that the only reason to assume that $G$ is locally finite.in the case of positive characteristic was to meet the assumptions under which Theorem $A$ could be 
used. Thus the assertion of Theorem 2 also holds for arbitrary semigroups if $K$ is not algebraic over its prime subfield (cf. [8, Theorem 3]).

Theorem 2 may be also obtained using Burnside's theorem on irreducible semigroups of matrices (cf. [2]). This may be done through Corollary 1, Theorem 1 and some considerations which are quite different from those presented here.

3. Main theorems. Before stating the main theorems we will prove the following lemma.

Lemma 3. Let $K$ be an infinite field and A a $K$-algebra. Assume there exists $n \geqslant 1$ such that for any $a \in A$ there is a subalgebra $B_{a}$ in $A$ with $a \in B_{a}$ and $n_{B_{a}} \leqslant n$. Then $A$ is semilocal and $n_{\mathrm{A}} \leqslant n$.

Proof. It is easy to see that we may assume that $A$ contains a unity 1 and $1 \in B_{a}$ for any $a \in A$. Then $\sigma_{A}(a)=\{\lambda \in K \mid \lambda-a$ is not invertible in $A\} \subset\{\lambda \in K \mid \lambda-a$ is not invertible in $\left.B_{a}\right\}=\sigma_{B_{a}}(a)$. Since $\left|\sigma_{B_{a}}(a)\right| \leqslant n_{B_{a}} \leqslant n$ (cf. [7]), $\left|\sigma_{A}(a)\right| \leqslant n$. It follows from [7, Theorem 3], that $A$ is semilocal and $n_{\mathrm{A}} \leqslant n$.

The above lemma does not hold in the case of a finite field $K$. For example put $A=\bigoplus_{i=1}^{\infty} K_{i}, K_{i}=K$ for $i=1,2, \ldots$ However, it may be checked that the assertion holds if we assume that for any finite set of elements $a_{1}, \ldots, a_{r} \in A$ there exists a subalgebra $B$ in $A$ with $a_{1}, \ldots, a_{r} \in B$ and $n_{B} \leqslant n$.

Now we are ready to prove our main result for fields of characteristic zero.

THEOREM 3. Let ch $K=0$. Then $K[G]$ is semilocal if and only if

(i) $G$ is locally finite and there exists $N \geqslant 1$ such that $G$ has no subgroup of order exceeding $N$, and

(ii) $E(G)=\bigcup_{i=1}^{s} E_{i}$ for some disjoint subsemigroups $E_{i}$ with the property that if $e, f \in E_{i}$ and $g \in G$ then ege is invertible in $e G e$ if and only if so are the elements efge and egfe-and then ege $=$ efge $=$ egfe.

Proof. In view of Corollary 1 we may assume that $K$ is algebraically closed.

Necessity. $G$ is locally finite by Theorem B. If $H \subset G$ is a subgroup, then $K[H] \simeq$ $K[H] /(K[H] \cap J(K[G])) \subset K[G] / J(K[G])$ since $K[H] \cap J(K[G]) \subset J(K[H])=0$ by [10]. Thus $|H| \leqslant \operatorname{dim}_{K} K[G] / J(K[G])$ and by Theorem 1 it is enough to put $N=\operatorname{dim}_{K} K[G] /$ $J(K[G])$.

From Theorem 2 it follows that $E\left(G / \sim_{K}\right) \subset G / \sim_{K}$ is finite. Let $E_{1}, \ldots, E_{s}$ be all the classes of the congruence $\sim_{K}$ in $E(G)$. If $g \in G$ and $e, f \in E_{i}$ for some $i$, then $e-f \in$ $J(K[G])$ and so ege-efge $\in J(K[G]) \cap K[e G e]=J(K[e G e])$. Assume that ege is invertible in $e G e$. Then $(e g e)^{k}=e$ for some $k \geqslant 1$ and $\left(e-(e f g e)^{k}\right) \in J(K[e G e])$. Since $J(K[e G e])$ is nil ideal, $\left(e-(e f g e)^{k}\right)^{n}=0$ for some $n \geqslant 1$ and there exists $r \geqslant 1$ such that $(e f g e)^{r}=e$. Thus ege $-e f g e \in J(K[e G e]) \cap K\left[(e G e)_{1}\right] \subset J\left(K\left[(e G e)_{1}\right]\right)=0$ and ege = efge. Similarly ege = egfe. 
If we assume that egfe is invertible in $e G e$, then, as above, ege is also invertible in eGe and $e g e=e f g e=e g f e$. In particular, $e f e=e$ and so the sets $E_{i}$ are semigroups.

Sufficiency. Let us notice that the assumptions on $G$ are inherited by any subsemigroup $H$. The number of nonempty sets $H \cap E_{i}$ will be thus denoted by $s_{H}$. We will prove that there exists a common bound (dependent on $N$ and $s$ ) on dimensions of $K$-algebras $K[H] / J(K[H])$ for finitely generated, and hence finite, subsemigroups $H$ in $G$. Then the assertion will follow from Lemma 3 . It will be done in two steps.

Step I. There exists a bound on the number $a_{H}$ of maximal ideals of $K[H]$. Let us notice first that if $e, f \in E_{i} \cap H$ for some $i$, then these elements belong to the same ideals in $K[H]$. Moreover, for any maximal ideal $M$ in $K[H]$ there exists $e \in E(H)$ with $e \notin M$ (see the proof of Theorem 1). Thus we easily get:

$$
\sum_{i=1}^{s} a_{e_{i} H e_{i}} \geqslant a_{H}, \quad \text { where } e_{i} \text { is a representative from } E_{i} \text {. }
$$

Assume $s_{H}=1$. Then $(e H e)_{0}=\varnothing$ or $(e H e)_{0}$ is a nil ideal in $e \mathrm{He}$ if $e \mathrm{He}$ is a semigroup with zero. Since $H$ is finite, then $\omega=\left\{\sum \lambda_{i} g_{i} \in K\left[(e H e)_{0}\right] \mid \sum \lambda_{i}=0\right\}$ is nilpotent and so $\omega \subset J(K[e H e])$. Now $\left|(e H e)_{1}\right| \leqslant N$ and hence $\operatorname{dim}_{K} K[e H e] / J(K[e H e]) \leqslant N+1$. Thus $K[e H e]$ has at most $N+1$ maximal ideals which implies $a_{H} \leqslant a_{e H e} \leqslant N+1$.

If $H$ is an arbitrary finite subsemigroup in $G$, then

$$
a_{(e \mathrm{He})_{0}}+N \geqslant a_{e H e} \text { for any } e \in E(H) \text {. }
$$

Moreover, it is easily seen that $E_{i} \cap(e \mathrm{He})_{0}=\varnothing$ if $e \in E_{i}$. Hence the number $s_{(e \mathrm{H})_{0}}$ of nonempty sets $E_{i} \cap(e H e)_{0}$ is less than $s_{H}$. Thus, in view of $(*)$ and $(* *)$, the proof may be completed by induction.

Step II. There exists a bound on dimensions of simple images of $K[H]$. Let $A$ be a simple ring and let $\varphi: K[H] \rightarrow A$ be an epimorphism. Since $K$ is algebraically closed and $|H|<\infty, A \simeq M_{m}(K)$ for some $m \geqslant 1$. We may assume that there is no finite subsemigroup $H^{\prime}$ in $G$ with $s_{H^{\prime}}<s_{H}$ and such that $M_{m}(K)$ is an image of $K\left[H^{\prime}\right]$.

Let $i \in\{1, \ldots, s\}$. We will first show that $\varphi(e)=\varphi(f)$ for $e, f \in E_{i}$. It may be assumed that $\varphi(e)=\left[\begin{array}{ll}l & 0 \\ 0 & 0\end{array}\right]$ where $I$ is the unity of $M_{\operatorname{tr\varphi }(e)}(K)$. Then $\varphi(f)=\left[\begin{array}{cc}I & A_{f} \\ B_{f} & B_{f} A_{f}\end{array}\right]$ for some rectangular matrices $A_{f}, B_{f}$ since $e f e=e$ and $f e f=f$.

Let $F$ be the ideal in $H$ generated by $(e H e)_{0}$. Then $e(H / F) e=(e(H / F) e)_{1} \cup\{\theta\}$ where $\theta$ is the zero element of $e(H / F) e$. Since $\varphi(K[F]) \triangleleft \varphi(K[H])=M_{m}(K)$ and $s_{F}<s_{H}$, we have $\varphi(K[F])=0$. Thus $\varphi(K[H])=\bar{\varphi}(K[H / F])$ for a homomorphism $\bar{\varphi}$ such that $\varphi=\bar{\varphi} \psi$ and $\psi: K[H] \rightarrow K[H / F]$ is a natural homomorphism. Hence $H$ may be replaced by $H / F$ in our considerations and so we may assume that the zero is the only non-invertible element in $e H e$. Now the assumption (ii) means that $e g e=e g f e=e f g e$ for any $g \in H$.

If $g \in H$ then $\varphi(g)$ may be written in the form $\left[\begin{array}{cc}u_{\mathrm{g}} & A_{\mathrm{g}} \\ B_{\mathrm{g}} & C_{\mathrm{g}}\end{array}\right]$, where $u_{\mathrm{g}} \in M_{\mathrm{tr} \varphi(e)}(K) \cap$ $\varphi(e H e)$. The condition $\varphi($ ege $)=\varphi($ efge $)$ implies $u_{\mathrm{g}}=u_{\mathrm{fg}}=u_{\mathrm{g}}+A_{f} B_{\mathrm{g}}$, and so $A_{f} B_{\mathrm{g}}=0$. Since $\varphi(H)$ is an irreducible subsemigroup in $M_{m}(K), A_{f}=0$. Similarly $B_{f}=0$, which yields $\varphi(e)=\varphi(f)$. 
Thus we have shown that $\varphi(H)$ has at most $s$ idempotents. Since $\varphi(e) \varphi(H) \varphi(e)=$ $\varphi(e \mathrm{He}) \subset \varphi\left((e \mathrm{He})_{1}\right) \cup\{0\}$, it is enough to prove the following lemma in order to establish Step II.

Lemma 4. Let $H \subset M_{m}(K)$ be a torsion irreducible semigroup. If $H$ has at most $s$ nonzero idempotents and $\left|(e H e)_{1}\right| \leqslant N$ for some $0 \neq e \in E(H)$, then $m \leqslant N(2 s+1)$.

Proof. As above, we may assume that $e=\left[\begin{array}{ll}I & 0 \\ 0 & 0\end{array}\right], H$ contains the zero matrix, $e H e=(e H e), \cup\{0\}$, and any element $g \in H$ has a representation of the form $\left[\begin{array}{ll}u_{\mathrm{g}} & A_{\mathrm{g}} \\ B_{\mathrm{g}} & C_{\mathrm{g}}\end{array}\right]$ for some invertible or zero matrix $u_{\mathrm{g}} \in M_{\mathrm{tre}}(K)$.

If $u_{\mathrm{g}} \neq 0$, then $(e \mathrm{~g})^{n}=\left[\begin{array}{cc}u_{\mathrm{g}} & A_{\mathrm{g}} \\ 0 & 0\end{array}\right]^{n}=\left[\begin{array}{cc}I & u_{\mathrm{g}}^{-1} A_{\mathrm{g}} \\ 0 & 0\end{array}\right]$ is an idempotent in $H$ for some $n \geqslant 1$. Thus, by the hypothesis, we have at most $N s$ possibilities for such products eg. Since the set $\left\{A_{\mathrm{g}}\right\}_{\mathrm{g} \in H}$ generates a $K$-space of dimension $(m-\operatorname{tr} e) \operatorname{tr} e$, then we get at least ( $m-$ $\operatorname{tr} e) \operatorname{tr} e-N s$ nonzero elements of the form $\left[\begin{array}{cc}0 & A_{\mathrm{g}} \\ 0 & 0\end{array}\right]$ in $H$. Now $M_{m}(K)$ has no nonzero nil ideals and so any left ideal in $H$ contains a non-nilpotent element.

Since $H$ is torsion, for any $e g=\left[\begin{array}{cc}0 & A_{g} \\ 0 & 0\end{array}\right] \in H \backslash\{0\}$ there exists $h \in H$ such that heg is a nonzero idempotent. Assume that $h\left[\begin{array}{cc}0 & A_{\mathrm{g}} \\ 0 & 0\end{array}\right]=h^{\prime}\left[\begin{array}{cc}0 & A_{\mathrm{g}^{\prime}} \\ 0 & 0\end{array}\right]$ is non-zero idempotent for some $h, h^{\prime}, g, g^{\prime} \in H$ with $u_{\mathrm{g}}=u_{\mathrm{g}^{\prime}}=0$. Then $u_{\mathrm{h}} A_{\mathrm{g}}=u_{\mathrm{h}^{\prime}} A_{\mathrm{g}^{\prime}}$ and $B_{\mathrm{h}} A_{\mathrm{g}}=B_{\mathrm{h}^{\prime}} \cdot A_{\mathrm{g}^{\prime}}$. If $u_{\mathrm{h}} \neq 0$, then $A_{\mathrm{g}}=u_{\mathrm{h}}^{-1} u_{h^{\prime}} A_{\mathrm{g}^{\prime}}$. If $u_{\mathrm{h}}=0$, then $u_{\mathrm{h}^{\prime}}=0$ and $u_{\mathrm{gh}} A_{\mathrm{g}}=A_{\mathrm{g}} B_{\mathrm{h}} A_{\mathrm{g}}=A_{\mathrm{g}} B_{\mathrm{h}^{\prime}} A_{\mathrm{g}^{\prime}}=u_{\mathrm{g} h^{\prime}} A_{\mathrm{g}^{\prime}}$. Since $\left(B_{h} A_{g}\right)^{2}=B_{h} A_{g} \neq 0$, because $h\left[\begin{array}{cc}0 & A_{g} \\ 0 & 0\end{array}\right] \neq 0$, we have $u_{g h} \neq 0$. Thus $A_{g}=u_{g h}^{-1} u_{g h} \cdot A_{g^{\prime}}$. Hence in both cases $A_{\mathrm{g}}=v A_{\mathrm{g}}$, for some $v \in(e \mathrm{He})_{1}$, and so the same idempotent as from $\left[\begin{array}{cc}0 & A_{\mathrm{g}} \\ 0 & 0\end{array}\right]$ may be obtained by left multiplication from at most $N$ different elements $\left[\begin{array}{cc}0 & A_{\mathrm{g}^{\prime}} \\ 0 & 0\end{array}\right]$. It follows that $((m-\operatorname{tr} e) \operatorname{tr} e-N s) / N \leqslant s$ and hence $m \leqslant(2 s+1) N$ since $\operatorname{tr} e \leqslant$ $N$. This completes the proof of Step II and so the proof of the theorem.

By slight modifications of the above reasoning one can easily obtain the following result for positive characteristics.

THEOREM 4. Let ch $K=p>0$ and let $G$ be locally finite. Then $K[G]$ is semilocal if and only if

(i) there exists $N \geqslant 1$ such that any subgroup in $G$ has a p-subgroup of index not exceeding $N$, and

(ii) $E(G)=\bigcup_{i=1}^{s} E_{i}$ for disjoint sets $E_{i}$ with the property that if $e, f \in E_{i}$ and $g \in G$, then 
ege is invertible in eGe if and only if so are efge and egfe-and then ege, efge and egfe are in the same coset of some normal p-subgroup in $(e G e)_{1}$.

4. Special cases. Let us mention some particular cases in which the obtained description simplifies considerably. As an easy consequence of Theorems 3 and 4 we obtain the following result.

COROLlary 2. Let $G$ be commutative. Then $K[G]$ is semilocal if and only if $G$ is torsion with $E(G)$ finite and

(i) $G$ has no infinite subgroups if ch $K=0$, and

(ii) any subgroup in $G$ contains a $p$-subgroup of finite index if ch $K=p>0$.

In [5] the problem of characterising the local semigroup algebras was raised; even in the case of group algebras the complete answer is not known. Here we can prove the following result.

THEOREM 5.

(i) If ch $K=0$, then $K[G]$ is local if and only if $G$ is locally finite and $e G e=\{e\}$ for any $e \in E(G)$.

(ii) Assume $G$ is locally finite and ch $K=p>0$. Then $K[G]$ is local if and only if $e G e$ is a p-group for any $e \in E(G)$.

Proof. Assume that $K[G]$ is local. Then any $e \in E(G)$ is a minimal idempotent and so $e G e$ is a group since $G$ is torsion. Thus the necessity follows as in Theorems 3 and 4 .

In view of Lemma 3 , to prove sufficiency it is enough to show that $K[H]$ is local for any finite subsemigroup $H$ in $G$. We may choose a $K$-basis for the augmentation ideal in $K[H]$ consisting of elements of the forms $e-g$ and $e-f$ where $e, f \in E(H)$ and $g^{k}=e$ for some $k \geqslant 1$. Thus it is enough to prove that all such elements are nilpotent. For $e$ and $g$ as above we have $(e-g)^{k} \in K[e H e]$. Since $K[e H e]$ is local (by [10, Lemma 8.1.17]) and locally finite, $(e-g)^{k}$ is nilpotent. Thus $e-g$ is also nilpotent. For $e, f \in E[H]$ put $x=e f e$ and $y=f e f$. It may be easily checked that $(e-f)^{2 n+1}=(e-x)^{n}-(f-y)^{n}$ for any $n \geqslant 1$. Now $x^{r}=e$ and $y^{s}=f$ for some $r, s \geqslant 1$ and hence, as above, $e-f$ is nilpotent. This completes the proof.

AdDED IN PROOF. Recently, we have realized that the theory of completely 0-simple semigroups may be used to simplify the proof of the sufficiency in Theorems 3 and 4 .

\section{REFERENCES}

1. A. H. Clifford and G. B. Preston, The algebraic theory of semigroups (American Mathematical Society, 1961).

2. C. Faith, Algebra II: Ring Theory (Springer-Verlag 1976).

3. J. Krempa and J. Okniński, Semilocal, semiperfect and perfect tensor products, Bull. Acad. Polon. Sci. Sér. Sci. Math. 28 (1980), 249-256.

4. J. Lawrence and S. M. Woods, Semilocal group rings in characteristic zero, Proc. Amer. Math. Soc. 60 (1976), 8-10. 
5. Sin-Min Lee, A condition for a semigroup ring to be local, Nanta Math. 11 (1978), 136-138.

6. W. D. Munn, On semigroup algebras, Proc. Cambridge Philos. Soc. 51 (1955), 1-15.

7. J. Okniński, On spectrally finite algebras, Bull. Acad. Polon. Sci. Sér. Sci. Math. 27 (1979), 515-519.

8. J. Okniński, Spectrally finite and semilocal group rings, Comm. Algebra 8 (1980), 533-541.

9. J. Okniński, Artinian semigroup rings, Comm. Algebra 10 (1982), 109-114.

10. D. S. Passman, The algebraic structure of group rings. (Interscience, 1977).

11. E. I. Zelmanov, Semigroup algebras with identities, Siberian Math. J. 18 (1977), 787-798.

UNIVERSITY OF WARSAW,

00-901 WARSAW,

POLAND. 